\title{
Application of a $\mathrm{H}_{2}^{+}$-like model to helium atom. Solution of the wave equation at the ground state
}

\author{
Feng Xu \\ Department of Biological Chemistry, University of Michigan Medical School, Ann Arbor, \\ MI 48109-0606, USA
}

Received 17 November 1988; final version 18 January 1989

The wave equation of helium atom is solved based on a " $\mathrm{H}_{2}^{+}$-like" model. A good agreement between the theoretical and experimental ionization energies is obtained.

PACS: 31.20

The wave equation of hydrogen molecule ion $\mathrm{H}_{2}^{+}$, in which one electron moves in the field of two oppositely charged nuclei, can be exactly solved [1]. Adequate numerical informations about its electronic wave function $\psi$ and corresponding eigen energy $E_{a}$ (attractive interaction) has been given [1c]. These results can be modified to apply to more generalized systems, in which the charges of three particles are $Z^{*}$ and $Z\left(|Z|,\left|Z^{*}\right| \geqq 1 ; Z Z^{*}<0\right)$, as shown in Fig. 1 . All that is necessary is to multiply the internuclear distance column by $\left(\left|Z Z^{*}\right|\right)^{-1}$ and the energy column by $\left(Z Z^{*}\right)^{2}$ in the tables of Bates et al. [1c]. The two

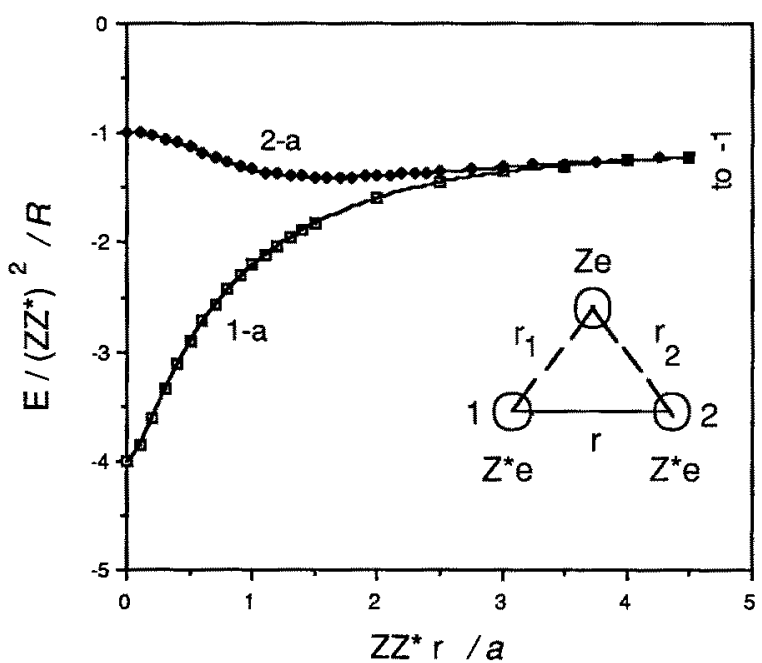

Fig. 1. " $\mathrm{H}_{2}^{+}$-like" three-body system $\left(Z Z^{*}<0\right)$ and its potential energy (attractive contribution) as function of $r$ for the ground state $(1-a)$ and the first excited state $(2-a)$. Curves made according to the data from $[1 \mathrm{c}]\left(1 s \sigma_{g}, 2 p \sigma_{u}\right)$. $R$ : Rydberg; $a$ : Bohr radius
$E_{a}$ which belong to the ground and the first excited states of He atom discussed later are shown in Fig. 1 as function of the interfocal distance $r$. The total potential energy of the system is the sum of $E_{a}$ plus $E_{r}$, the repulsive contribution due to the interaction between the two particles at the foci.

$\mathrm{He}$ atom comprises also three particles, but now the two with the same sign of charge are electrons. Is it possible to apply a " $\mathrm{H}_{2}^{+}$-like" model to $\mathrm{He}$ atom?

Nuclei are much heavier than electrons. It looks much like that in an atom it is the electrons who move around the nucleus. But we know that the essential things in the electronic motion are the relative coordinates of electrons with respect to the nucleus. The choice of the origin of coordinates, whether on the nucleus or on one of the electrons, does not affect the final results about wave function $\psi$ and eigen energy $E$. For example, we can have a wave equation which describes the "relative motion" of the nucleus with respect to the electron in $\mathrm{H}$ atom if the latter carries the origin of coordinates. Presented in appropriate "electronic units", this wave equation will be in the same form as that in familiar atomic units. Solving our wave equation and converting $E$ obtained to atomic units, we shall have the same results about the potential energy levels of $\mathrm{H}$ atom.

Based on the same idea, we now consider He atom in the coordinates shown in Fig. 1 with the two electrons on the foci 1,2 , separated by the distance $r$. Now $Z^{*}=-1, Z=+2$. The relative positions of the nucleus with respect to the electrons are given by $r_{1}, r_{2}$, and an angle parameter. Taking into account the difference of charge, the tables of Bates et al. [1 c] 


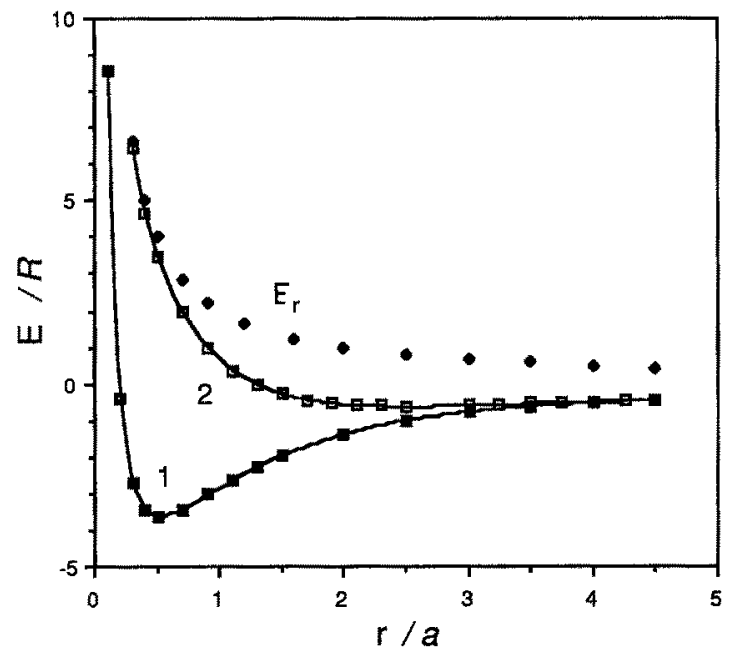

Fig. 2. Potential energy $E\left\{=\left(E_{a}+E_{r}\right)-E_{a}\right.$ (at $\left.\left.r=\infty\right)\right\}$ of helium atom as function of $r$ for: (1) ground state, (2) first excited state. $E_{r}:$ repulsive contribution

allow us to obtain $\psi(n)(n=0,1, \ldots$, for the ground, first excited, ... states) as function of $r$. For the convenience, all our results will be presented in atomic units.

When $Z^{*}=-1, Z=+2$, the curve $1-a$ in Fig. 1 represents the $E_{a}$ of the ground state in $\mathrm{He}$ atom. At $r=0, E_{a}=-16$. At $r=\infty, E_{a}=-4$. When the two electrons come together, the system will become a "super H atom" consisted of one nucleus $\left(Z_{+}=+2\right)$ and one "super electron" $\left(Z_{-}=-2\right)$. When one electron is separated away, the system will become $\mathrm{He}^{+}\left(Z_{+}=+2, Z_{-}=-1\right)$. Since the ground state potential energy of "H atom-like" systems is given by $E=-\left(Z_{+} Z_{-}\right)^{2}$, it is not surprising that we have these limiting $E_{a}$ values.

Having added the repulsive energy $E_{r}(=2.000 / r)$ between two electrons, the total energy $E_{a}+E_{r}$ for the ground state as function of $r$ is shown in Fig. 2 (curve 1). The minimum $E(0)=-7.614$ is at $r(0)=0.5$.

Up to now the two electrons are assumed to be identical. Since only one of the two possible spin configurations is permitted in the same quantum state, the observable ionization potential (IP) for $\mathrm{He}$ atom is $-7.614 / 2+4.000 / 2=1.807$ or $24.587[\mathrm{eV}]$, which equals to the experimental value [3].

As shown for $\mathrm{H}_{2}^{+}, \psi(0)$ is symmetric to the two centers [1 c]. For He atom, the "appearance" of the nucleus in the near space around electrons is symmetric. In other words, the electronic $\psi_{e}(0)$ at the ground state must be spherically symmetric to the nucleus. $r(0)$ only has statistical sense. Since the two repulsive electrons tend to keep away from one another as far as possible, $r(0)=0.5$ indicates that the maximum of the radial part of $\left[\psi_{e}(0)\right]^{2}$ is at $r(0) / 2=0.25$.
As shown in Fig. 2, the next minimum potential energy $E(1)=-4.618$ at $r(1)=2.5$ (curve 2). Having considered Pauli principle, the energy required for an electron jumping from $E(0)$ to $E(1)$ is $7.614 / 2-4.618$ / $2=1.498$ or $20.38[\mathrm{eV}]$. The experimental results are: $\left(1 \mathrm{~s}^{2}\right)-(1 s 2 s): \quad 19.81 \sim 20.61 \mathrm{eV} ; \quad\left(1 s^{2}\right)-(1 s 2 p)$ : $20.96 \sim 21.21 \mathrm{eV} \mathrm{[3]}$.

At the first excited state, $\psi(1)$ is symmetric to a nodal plane between two foci $\left([1 \mathrm{c}], 2 p \sigma_{u}\right)$. Thus the corresponding electronic $\psi_{e}(1)$ shows spherical symmetry with respect to the nucleus but with a nodal cosphere inside, which is similar to the $\psi_{e}(2 s)$ of $\mathrm{He}^{+}$.

We may extend our treatment to other two-electron contained systems, e.g. $\mathrm{Li}^{+}, \mathrm{Be}^{2+}, \mathrm{B}^{3+}, \mathrm{C}^{4+}$. Using the tables of Bates et al. [1 c], the calculated IP (and $r(0)$ ) are: $\mathrm{Li}^{+}: 78.097(0.27) ; \mathrm{Be}^{2+}: 164.324(0.15)$; $\mathrm{B}^{3+}: 285.103(0.12) ; \mathrm{C}^{4+}: 437.753(0.1)$. These values are, however, slightly higher $\left(\mathrm{Li}^{+}: 3 \% ; \mathrm{Be}^{2+}: 7 \%\right.$; $\mathrm{B}^{3+}: 10 \% ; \mathrm{C}^{4+}: 12 \%$ ) than the experimental results [3]. When $Z$ goes up, $r(0)$ becomes smaller and smaller. At very close distance, our treatment on the coulombic and Pauli repulsive interactions between two electrons might be too simplified.

By iteration, quantum mechanic approximation theories have given better results on IP of $\mathrm{Li}^{+}, \mathrm{Be}^{2+}$, $\mathrm{B}^{3+}, \mathrm{C}^{4+} \ldots$ systems [4]. Considering its simplicity, our model is qualitatively quite satisfactory [5]. Usually nuclei are considered immobile and wave equations are set up on the coordinates of electrons. The discussion made above suggests that sometimes a change of coordinates might be helpful in the study of some specific multi-electron systems.

\section{References}

1. a) Burrau, O.: K. Dan. Vidensk. Selsk. 7 (14) 1 (1927)

b) Slater, J.C.: Quantum theory of molecules and solids. Vol. 1, pp. 1-19. New York, San Francisco, Toronto, London: McGrawHill 1963

c) Bates, D.R., Ledsham, K., Stewart, A.L.: Philos. Trans. R. Soc. London 246, 215 (1953)

2. The values of the universal physical constants and the energy conversion factors used are taken from: Handbook of Chemistry and Physics: 69th Edn. pp. 192-195. Boca Raton (Florida): CRC 1988

3. a) Ref. 2, pp. E-78

b) Moore, C.E.: Atomic Energy Levels. 35/Vol. 1, pp. 4-5. Nat. Bur. Stand. (U.S.) 1971

4. a) Flygare, W.H.: Molecular structure and dynamics, Chap. 5. Englewood Cliffs: Prentice-Hall 1978

b) Pekeris, C.L.: Phys. Rev, 115, 1216 (1959)

5. Since $\mathrm{H}_{2}^{+}$is a diatomic system, the nuclear vibrational and rotational energy contributions, in which the nuclear mass plays important role, should be taken into account [1]. He atom (as well as $\left.\mathrm{Li}^{+}, \mathrm{Be}^{2+}, \mathrm{B}^{3+}, \mathrm{C}^{4+} \ldots\right)$ is monoatomic molecule whose kinetic part of energy does not appear in the Hamiltonian concerned here. The relative motion of the nucleus "around" the electrons is only a mathematic description after the origin of coordinates is set on the electrons 